\title{
The spin axes orbital alignment of both stars within the eclipsing binary system V1143 Cyg using the Rossiter-McLaughlin effect ${ }^{\star} \star \star$
}

\author{
S. Albrecht ${ }^{1}$, S. Reffert ${ }^{2}$, I. Snellen ${ }^{1}$, A. Quirrenbach ${ }^{2}$, and D. S. Mitchell ${ }^{3}$ \\ ${ }^{1}$ Leiden Observatory, Leiden University, PO Box 9513, 2300 RA Leiden, The Netherlands \\ e-mail: albrecht@strw.leidenuniv.nl \\ 2 ZAH - Landessternwarte, Königstuhl 12, 69117 Heidelberg, Germany \\ ${ }^{3}$ California Polytechnic State University, San Luis Obispo, CA 93407, USA
}

Received 25 May 2007 / Accepted 30 July 2007

\begin{abstract}
Context. The Rossiter-McLaughlin (RM) effect, a rotational effect in eclipsing systems, provides unique insight into the relative orientation of stellar spin axes and orbital axes of eclipsing binary systems.

Aims. Our aim is to develop a robust method to analyze the RM effect in an eclipsing system with two nearly equally bright components. This gives access to the orientation of the stellar rotation axes and may shed light on questions of binary formation and evolution. For example, a misalignment between the spin axes and the angular momentum of the system could bring the observed and theoretical apsidal motion into better agreement for some systems, including V1143 Cyg.

Methods. High-resolution spectra have been obtained both out of eclipse and during the primary and secondary eclipses in the V1143 Cyg system, using the $0.6 \mathrm{~m}$ Coudé Auxiliary Telescope (CAT) and the high-resolution Hamilton Echelle Spectrograph at the Lick Observatory. The Rossiter-McLaughlin effect is analyzed in two ways: (1) by measuring the shift of the line center of gravity during different phases of the eclipses and (2) by analysis of the line shape change of the rotational broadening function during eclipses.

Results. We measured the projection of the stellar rotation axes using the rotation effect for both main-sequence stars in an eclipsing binary system. The projected axes of both stars are aligned with the orbital spin within the observational uncertainties, with the angle of the primary rotation axis $\beta_{\mathrm{p}}=0.3 \pm 1.5^{\circ}$, and the angle of the secondary rotation axis $\beta_{\mathrm{s}}=-1.2 \pm 1.6^{\circ}$, thereby showing that the remaining difference between the theoretical and observed apsidal motion for this system is not due to a misalignment of the stellar rotation axes. Both methods utilized in this paper work very well, even at times when the broadening profiles of the two stars overlap.
\end{abstract}

Key words. stars: individual: V1143 Cyg - binaries: eclipsing - techniques: spectroscopic - methods: data analysis binaries: spectroscopic

\section{Introduction}

Eclipsing binaries are great stellar laboratories for gathering information on stellar surface structure. During eclipses, varying parts of the stellar disk are obscured, allowing the observer to gather spatially resolved information. Without eclipses, this information is difficult to access.

The crossing of a companion in front of a rotating star causes a change in the line profile of the eclipsed star as, for example, it first covers mainly the part of the stellar surface which is moving towards the observer. This change in the line profile results in a change in the center of gravity of the line and therefore in a change in the measured radial-velocity of the star. The strength and shape of this rotation effect is a function of the projection of the stellar axes on the sky, its inclination (for stars with differential rotation), the projected rotational velocity, the stellar radius, the radius of the companion, the stellar limb-darkening, and the orbital parameters of the system.

The rotation effect was first observed by Rossiter (1924) in $\beta$ Lyrae, and by McLaughlin (1924) in the Algol system. The

\footnotetext{
^ Based on observations obtained at the Lick Observatory, which is operated by the University of California.

$\star \star$ Appendix A is only available in electronic form at http://www. aanda.org
}

theory of the rotation effect is well understood (e.g. Kopal 1959; Hosokawa 1953; Ohta et al. 2005; Giménez 2006). In contrast, observations of the rotation effect in eclipsing binary systems are rare (e.g. Hube \& Couch 1982; and Worek 1996). Observation and analysis of the RM effect has recently received renewed interest, caused by the possibility of observing the spin-orbital alignment for transiting exoplanet systems (e.g. Queloz et al. 2000; and Winn et al. 2006) and the potential to observe features of the planetary atmosphere (Snellen 2004).

For the successful observation and interpretation of the rotation effect in a planetary system, the required $\mathrm{S} / \mathrm{N}$ and precision in radial velocity are significantly higher than those required to analyze the RM effect in a stellar binary system. However, the difficulty in analyzing a stellar binary system lies in the fact that one has to deal with the additional light from the eclipsing foreground star. The spectral lines of the two stars normally blend during the eclipses, which makes an analysis of the rotation effect in the framework of the change of the center of gravity during an eclipse difficult. Nevertheless, the observation of the rotation effect is of astrophysical interest in binary systems, as it might reveal the orientation of the stellar rotation axes and provide information about stellar surface velocity fields. The knowledge of these quantities might help to answer questions related to binary formation and evolution, and to the study of apsidal motion. 
Table 1. Parameters for V1143 Cyg taken from ESA (1997) $\ddagger$, Andersen

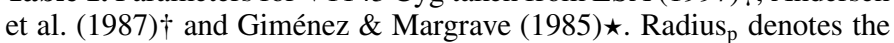
radius of the primary component and Radius $s_{\mathrm{s}}$ the radius of the secondary component. $L_{\mathrm{s}} / L_{\mathrm{p}}$ denotes the luminosity ratio between the secondary and primary.

\begin{tabular}{lll}
\hline \hline HIP & 96620 & \\
$\mathrm{RA}_{\mathrm{J} 2000}$ & $19^{\mathrm{h}} 38^{\mathrm{m}} 41^{\mathrm{s}}$ & $\ddagger$ \\
Dec $_{\mathrm{J} 2000}$ & $54^{\circ} 58^{\prime} 26^{\prime \prime}$ & $\ddagger$ \\
Parallax & $0^{\prime} .02512(56)$ & $\ddagger$ \\
$V_{\max }$ & $5.89 \mathrm{mag}$ & $\ddagger$ \\
Sp. Type & $\mathrm{F} 5 \mathrm{~V}$ & $\dagger$ \\
Period & $7^{\mathrm{d}} .6407568(6)$ & $\star$ \\
Inclination & $87.0(1)^{\circ}$ & $\dagger$ \\
Radius $_{\mathrm{p}}$ & $1.346(23) R_{\odot}$ & $\dagger$ \\
Radius $_{\mathrm{s}}$ & $1.323(23) R_{\odot}$ & $\dagger$ \\
$L_{\mathrm{s}} / L_{\mathrm{p}}$ & $0.96(3)$ & $\dagger$ \\
\hline
\end{tabular}

Accordingly, our aim in this research is twofold: I) to develop a method for deriving information about the orientation of the stellar rotation axes in an eclipsing system with two nearly equally bright components; II) to apply it to an astrophysically interesting system, V1143 Cyg (e.g. Andersen et al. 1987; Giménez \& Margrave 1985). V1143 Cyg (Table 1) is a bright system consisting of two F5V stars, and has a high eccentricity $(e=0.54)$ that makes it an ideal candidate for testing a new algorithm. Because of the high eccentricity, the spectral lines are not as extensively blended during eclipses. V1143 Cyg is a young $\left(2 \times 10^{9} \mathrm{yr}\right)$ system (Andersen et al. 1987), and the measured apsidal motion, i.e. the precession of the orbit in its own plane $(0.000705 \pm 0.000041 \%$ cycle, Giménez \& Margrave $1985)$, is only marginally compatible with what is expected theoretically $(0.00089 \pm 0.00015 \%$ cycle, Andersen et al. 1987). The precession of the periastron is caused by a general relativistic effect and a Newtonian contribution, the latter consisting of two terms which are due to the deformation of the two stars by tides and stellar rotation. Andersen et al. (1987) suggested the possibility that it could be possible that the tidal evolution has not yet achieved parallel rotation axes of the stars and the orbit. This would reduce the expected apsidal motion, thereby bringing it into a better agreement with the measured apsidal motion.

In the following section we present our observations. Section 3 describes the data reduction and the two methods used to derive the orbital and stellar parameters. The results are discussed in Sect. 4. A summary is given in Sect. 5.

\section{Observations}

V1143Cyg was observed in the summer/autumn of 2005 and 2006 with the $0.6 \mathrm{~m}$ CAT telescope at the Lick observatory, equipped with the high-resolution Hamilton Echelle Spectrograph. Observations of the primary eclipse $(\approx 4 \mathrm{~h})$ were made on the night of August 29/30, 2005 with two observations before, nine observations during, and four observations after the eclipse. The central part of the secondary eclipse $(\approx 8 \mathrm{~h})$ was observed during the nights August 04/05 and 27/28, 2005. In addition, another 20 observations were made out of eclipse in order to obtain an accurate orbit model. During each night, we also observed a set of different radial velocity standard stars. Before and after every exposure of V1143 Cyg ( $\approx 20 \mathrm{~min}$ ) or a standard star, we made a Thorium-Argon (ThAr) exposure to obtain a wavelength scale taken close in time to the observation, minimizing the influence of drifts in the spectrograph on our measurements. Data from the secondary eclipse taken on August 27/28 had to be discarded from the final analysis, because of an erroneous ThAr wavelength calibration during that night.

\section{Analysis and results}

For our analysis we used a wavelength range from $4430 \AA$ to $5750 \AA$, excluding the regions with telluric lines, $\mathrm{H} \beta$, and areas for which an insufficient continuum correction was achieved in the spectra (usually the edges of the orders). The first steps of the data reduction consisted of bad-pixel exclusion and continuum normalization. The latter was carried out by fitting a polynomial of 5 th order to the spectra and subsequently dividing the spectra by it.

In the next step, a weighted mean of the two wavelength scales, based on the two ThAr spectra obtained directly before and after each observation, was constructed. The weighting took into account time differences of the photon weighted midpoint of the observation and the times of the ThAr exposures. The resulting error in the calculated radial-velocity due to the drift of the spectrograph during the observation was estimated to be $75 \mathrm{~m} / \mathrm{s}$ on the basis of comparisons of several ThAr exposure pairs.

The Broadening Function (BF) was calculated to retrieve the line profiles of the two stars, governed by the velocity fields on the stellar surface and stellar rotation. The BF represents the function that projects a narrow-lined template onto the broadened spectrum. We calculated the BF using the singular value decomposition (SVD) technique. This approach is clearly described in Rucinski (1999), whereas the SVD algorithm itself can be found in Press et al. (1992) ${ }^{1}$. In order to calculate the $\mathrm{BF}$ one needs a narrow-lined template; we used the spectrum of the F7V star HD 222368 (Udry et al. 1999), obtained as one of the radial-velocity standard stars. Before using the spectrum of HD 222368 as a narrow-lined template we deconvolved it using the maximum-likelihood method in conjunction with a kernel calculated with the same code as applied in Sect. 3.2. An iterative approach was used to identify the kernel that gave the best results. The most satisfactory results are achieved when using a kernel with a projected rotational velocity $(v \sin i)$ of $5 \mathrm{~km} \mathrm{~s}^{-1}$. Figure 1 shows a part of a typical spectrum obtained from the V1143 Cyg system. It also shows for comparison the convolution of the narrow-lined template with the BF. As V1143 Cyg is a double-lined binary system with two components of the same spectral type, the BF itself consists of two peaks that represent the broadening functions of the two stars, shaped by the corresponding rotation and velocity fields.

In this study we are primarily interested in the data taken during the eclipses and their interpretation in the framework of the RM effect. Because of the high eccentricity $(e=0.54)$ of the system, the midpoints of the eclipses do not occur when the radial-velocities of the stars are equal, but shortly before and after the time of equal velocity, for the primary and secondary eclipses, respectively. During the middle and the end of the primary eclipse the lines of the two stars are blended. The same situation occurs during the beginning and the middle of the secondary eclipse. Hence, although the line blending is not as severe as in the case of a circular orbit, the light from the foreground star cannot be ignored.

We followed two different approaches to derive the spin axis of the eclipsed star with blended lines. The first method is

\footnotetext{
1 We took all singular values until the first derivation of the difference between the observed spectrum and the template broadened by the BF with respect to the singular values used for the calculation of the BF effectively reached zero.
} 


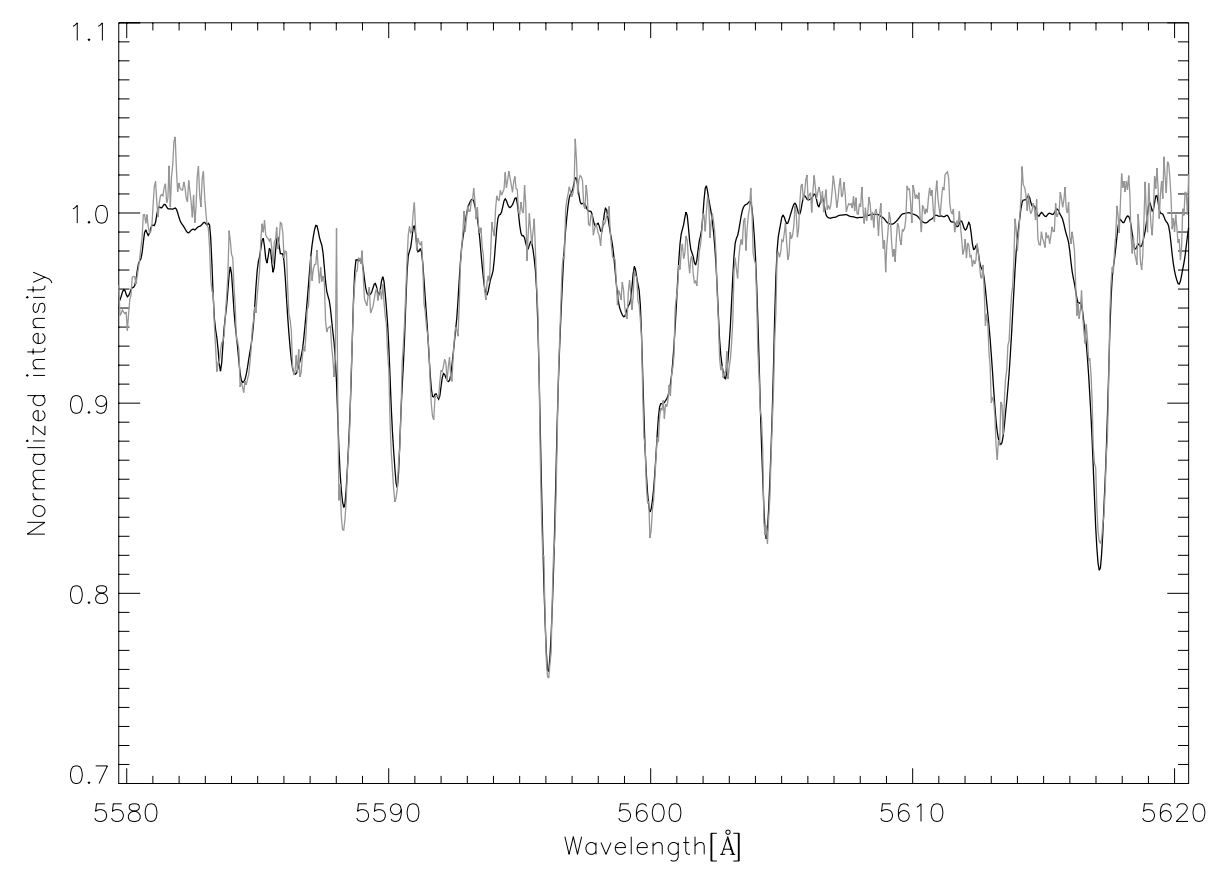

Fig. 1. Normalized spectrum of V1143 Cyg covering the wavelength range from 5580 to $5620 \AA$. The thin line represents the spectrum, and the thick line represents the convolution of the narrow-lined template with the BF obtained using the SVD algorithm. The quality of the representation of the spectrum by the BF and the template is also typical for other wavelength regions.

explained in Sect. 3.1. We first show how the influence of the foreground star is subtracted, and subsequently how the center of gravity from the BF of the eclipsed star is calculated. In Sect. 3.2 the BFs of both stars are used. Here we do not use the center of gravity of the measured BFs to derive the RM effect, but the shape of the BFs and their change during the eclipse. The measured BFs are compared to simulated BFs, and thereby the parameters that govern the rotation effect are derived.

\subsection{Method 1: the BF's center}

Before determining the parameters involved in the RM effect, an orbital model has to be obtained. To derive the radial-velocities of the components out of eclipse, we fitted two Gaussians to the two peaks in the BFs. A $\chi^{2}$ fit was applied to extract the orbital parameters. In this work, we adopted the orbital period $\left(7.6407568 \pm 6 \times 10^{-7}\right)$ for all fits, since it is derived at much higher accuracy from eclipse photometry than from radialvelocity variations (Giménez \& Margrave 1985). The inclination of the orbit $\left(87.0 \pm 0.1^{\circ}\right)$, and the sizes of the components $1.346 \pm 0.023 R_{\odot}$ and $1.323 \pm 0.023 R_{\odot}$ have been adopted from Andersen et al. (1987); these are needed for later analysis. The fitted parameters are shown in the second column of Table 2 with their 1- $\sigma$ uncertainties. In addition, the orbital parameters given by Andersen et al. (1987) and Giménez \& Margrave (1985) are shown for comparison in column five.

The tomography algorithm of Bagnuolo \& Gies (1991) is used to disentangle the primary and secondary spectra. This algorithm uses, the spectra obtained at different phases of the orbit, and the orbital parameters of the system, as input. It starts with two synthetic spectra without spectral lines for the two components in V1143 Cyg. For all observations taken outside the eclipses, it shifts the observed spectra in the rest-frames of each component using the newly obtained orbital parameters. Subsequently, the synthetic spectra are compared with the observed spectra. The mean of all the differences between the synthetic and observed spectra is added to the synthetic spectra. This complete process is repeated 50 times, but in our case it converged after only a few iterations.

In the next step, the spectrum of the foreground star is subtracted. For an observation out of eclipse this is straightforward, using the spectrum shifted in velocity space to the appropriate position. During eclipses, one has to incorporate the change in the light ratio of the two stars due to the eclipses. For this we assumed a linear limb-darkening law with a limb-darkening coefficient $(u)$ of 0.6 for both stars. Subsequently, the BF was calculated with only one star in the spectrum. Figures 2 and 3 show the BFs of the primary and secondary stars during their eclipses, after subtraction of the foreground star. Note that, for computational reasons, the continua of the observed spectra were set to zero and the signs of the spectra have been changed; this results in positive BFs.

The center of gravity of the absorption lines can now be extracted from the BFs and used to calculate the radial-velocity of the eclipsed star, including the radial-velocity anomaly introduced by the RM effect. Using the orbital model and the formula by Kopal $(1959)$ and Hosokawa $(1953)^{2}$ to calculate the rotation anomaly, the orbital parameters and the parameters relevant for the RM effect of the two stars can be derived jointly with a $\chi^{2}$ minimization algorithm; therefore $v \sin i$ and the angles $\beta_{\mathrm{p}}$ and $\beta_{\mathrm{s}}$ enter as new parameters. $\beta$ is the angle between the stellar spin axis projected onto the plane of the sky and the orbital spin axis projected onto the plane of the sky. $\beta=0^{\circ}$ would indicate a projected rotation axis perpendicular to the orbital plane, whereas $\beta=90^{\circ}$ would indicate that the rotation axis lies in the orbital plane. The longitude of the ascending node of the orbit $(\Omega)$ is not known, resulting in an ambiguity in the sign of the angle $\beta$. In our definition, a positive $\beta$ indicates that the RM effect, integrated over the complete eclipse, would give a positive residual in radial-velocity. In that case, the companion spends a longer time in front of the stellar surface moving towards us, than that

\footnotetext{
${ }^{2}$ Equation (5) has to be adopted for eccentric orbits.
} 


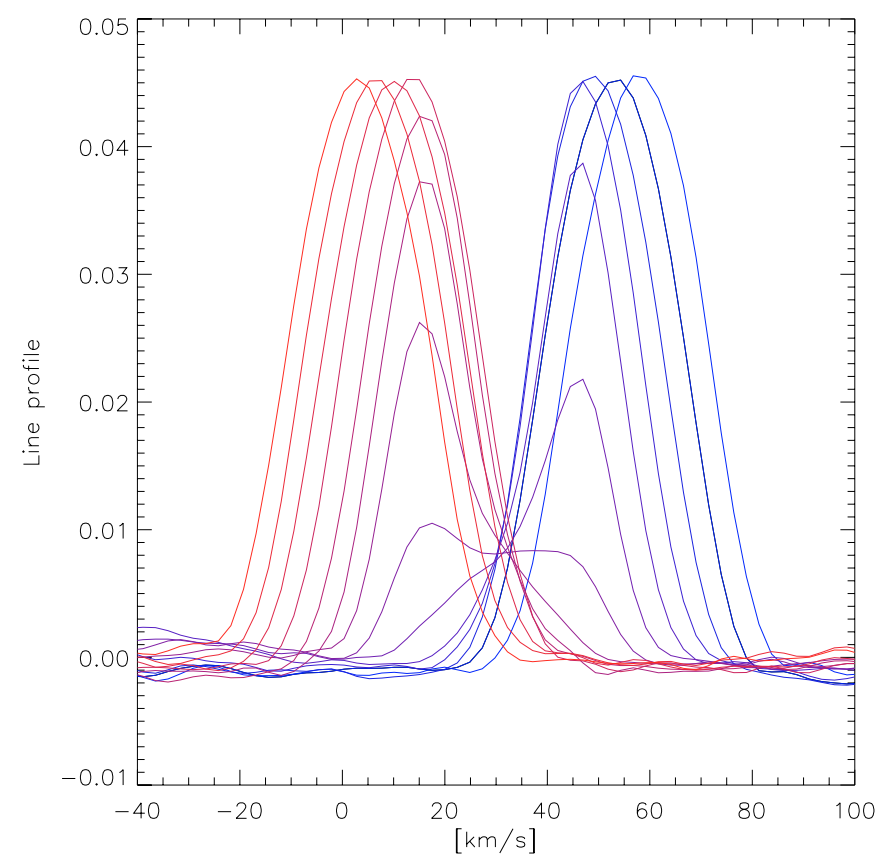

Fig. 2. The panel shows two BFs obtained shortly before (higher radialvelocities), nine during, and two after the primary eclipse (lower radialvelocities), with the secondary BF subtracted, in velocity space. One can see how the part of the stellar disk which is moving towards the observer is initially obscured. Later in the eclipse a more central part of the stellar disk is covered while at the end of the eclipse parts of the disk that emit red-shifted light due to the rotation are covered. Note that the $\mathrm{BF}$ of the primary is moving through velocity space. These observations were obtained at $\approx 30 \mathrm{~min}$ intervals.

part of the stellar surface that is moving away from us. The linear limb-darkening coefficients of both stars are fixed during the fits to 0.6 as they are only weakly constrained by our fits. In the $\chi^{2}$ fit, all data points out of eclipse and all data points during the primary and secondary eclipses are fitted simultaneously. Our radial-velocity data points and the best fit can be seen in Fig. 4 for the complete orbit including the two eclipses. Figure 5 shows the orbit from a point above the orbital plane. Figures 6 and 7 display the radial-velocities of the primary and secondary stars during and around their eclipses. The best fit parameters are listed in the third column of Table 2 with their 1- $\sigma$ uncertainties, as derived from the $\chi^{2}$ fit. All radial-velocity measurements are given with their epochs in Table A.1 in the Appendix. The radialvelocities directly after the primary eclipse, and the data point at a phase of 0.7 , have been extracted using a Gaussian fit to the BF of one star after the subtraction of the other star in the spectrum; however, there is no significant change in the derived parameters if these data points are omitted. The average uncertainties in the radial-velocities, out of the eclipses, are $0.15 \mathrm{~km} \mathrm{~s}^{-1}$ for the primary and $0.25 \mathrm{~km} \mathrm{~s}^{-1}$ for the secondary. The uncertainties of those radial-velocity measurements that were determined from the centers of the lines, we estimated to be $0.20 \mathrm{~km} \mathrm{~s}^{-1}$ for the primary and $0.32 \mathrm{~km} \mathrm{~s}^{-1}$ for the secondary. This was calculated by comparing them with the velocities obtained from a Gaussian fit outside the eclipses.

\subsection{Method 2: variation of the BF profile}

In our second approach, to derive information about the binary orbit and the orientation of the rotation axes, we simulated the shape of the BF of the two stars, as governed by the orbital

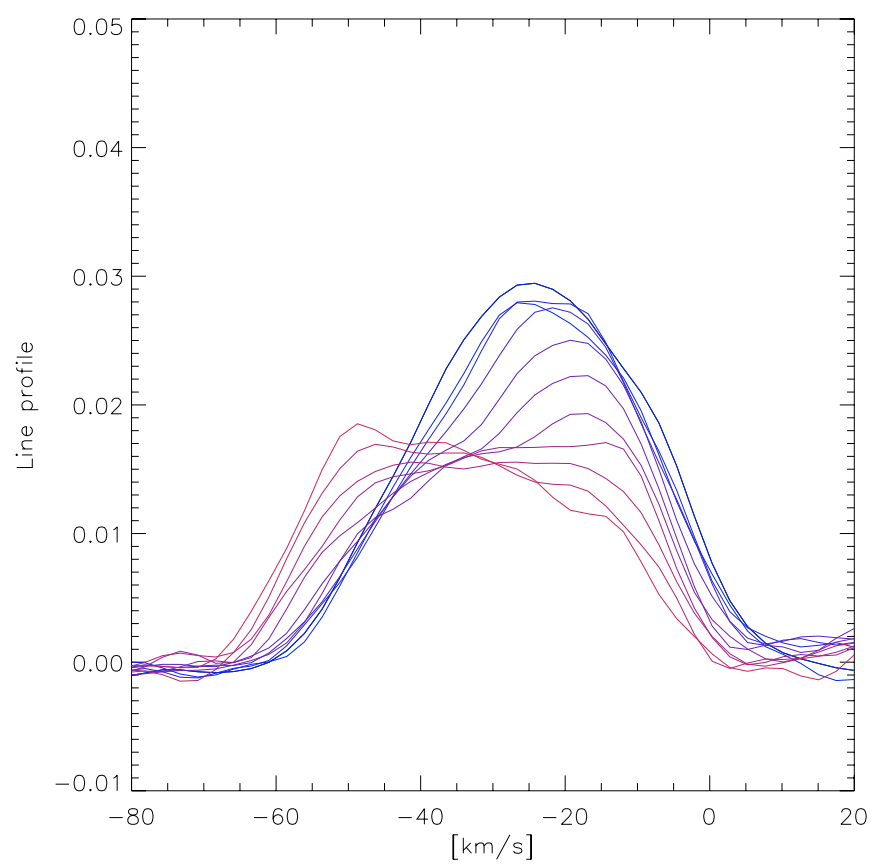

Fig. 3. The same as Fig. 2 but for the secondary eclipse. One can see BFs of the eleven observations obtained during the secondary eclipse with the line profile of the primary subtracted. The change in radialvelocity during the secondary eclipse is smaller than during the primary eclipse also, the coverage of the secondary is less than the coverage of the primary during its eclipse. This is due to the orbital inclination not being $90^{\circ}$, and the greater distance between the two stars along the line of sight during secondary eclipse (see Fig. 5).

motion, stellar rotation, orientation of the stellar spin axes, velocity fields on the stellar surface, limb-darkening and, in case of a measurement taken during an eclipse, the fraction of the stellar disk which is covered by the companion at the time of the measurement. These simulated BFs were subsequently compared to the measured BFs, and the relevant parameters determined. With this method, we not only use the first moment of the stellar absorption lines during the analysis, but we utilize the complete BF and its change over the course of the eclipse as a diagnostic tool for determining the orbital and stellar parameters.

We simulated the rotation profile of the two stars in the V1143 Cyg system with a few thousand elements across the visible half-spheres with equal surface brightness. We included linear limb-darkening, asymmetric macro-turbulence and solar-like differential rotation (See Gray 2005). Accordingly, besides the parameters which were fitted in Sect. 3.1, another parameter, the Gaussian width of the macro-turbulence, is required. We kept the $\sigma$ of the Gaussian for the tangential and radial-velocity fields $\zeta_{\mathrm{RT}}$ and their covered surface fraction equal, as the quality of the fit did not improve by including them as free parameters. The inclination of the orbit and the sizes of the two stars can be varied in our fits. However, in the final fits presented here, we used a linear limb-darkening coefficient of 0.6 , solid body rotation, and the inclination and radii from the literature (see also Sect. 4).

Each exposure of V1143 Cyg had a duration of $\approx 20 \mathrm{~min}$. Provided that one takes the photon midpoint of the observation, this poses no serious problem for the analysis of the center of gravity of the lines. However, looking at the line profile, one has to take two effects into account. First, the radial-velocities of the two stars change during a $20 \mathrm{~min}$ exposure. This effect is strongest in our data set at the time of the primary eclipse, where the change in radial-velocity is $\approx 3 \mathrm{~km} \mathrm{~s}^{-1}$ during one 


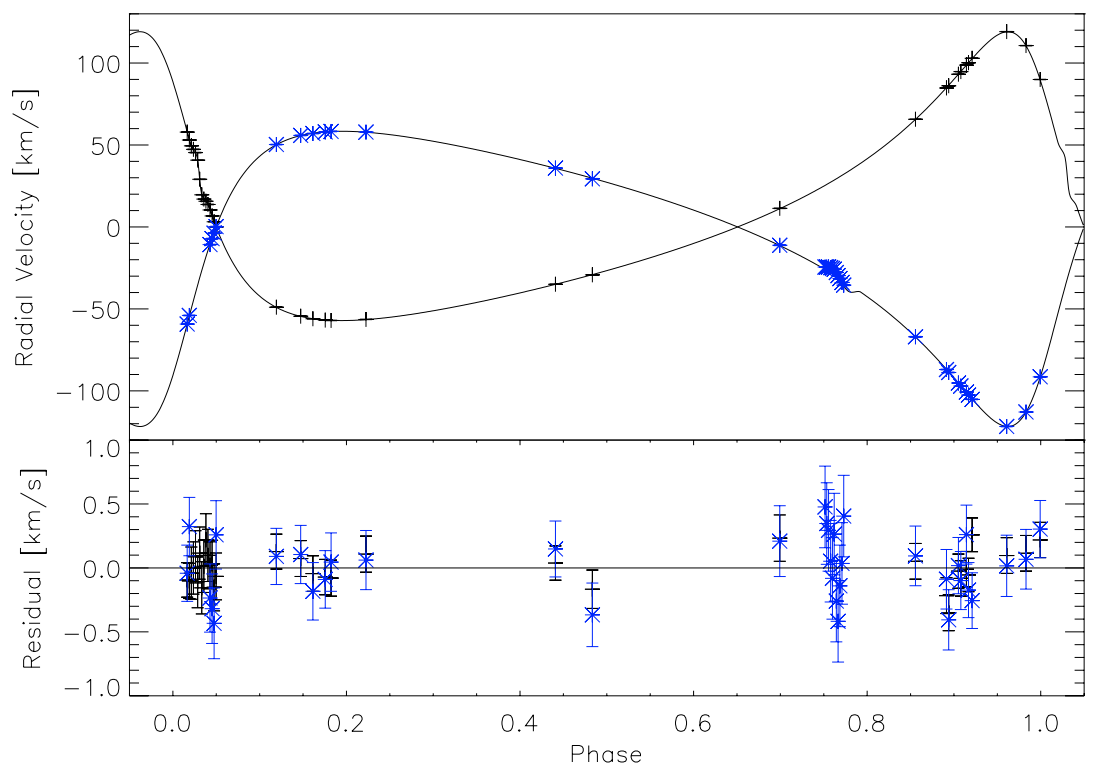

Fig. 4. The radial-velocity measurements of the primary and secondary components of V1143 Cyg, and the orbital solution, are plotted against orbital phase. The lower panel shows the difference between the best fit and the actual measurements of the radial-velocities of the primary and secondary component. The midpoint of the primary eclipse occurs at a phase of $\approx 0.03$, and the midpoint of the secondary eclipse at a phase of $\approx 0.77$. Please note that the systemic velocity in this graph and in Figs. 6 and 7 is already subtracted

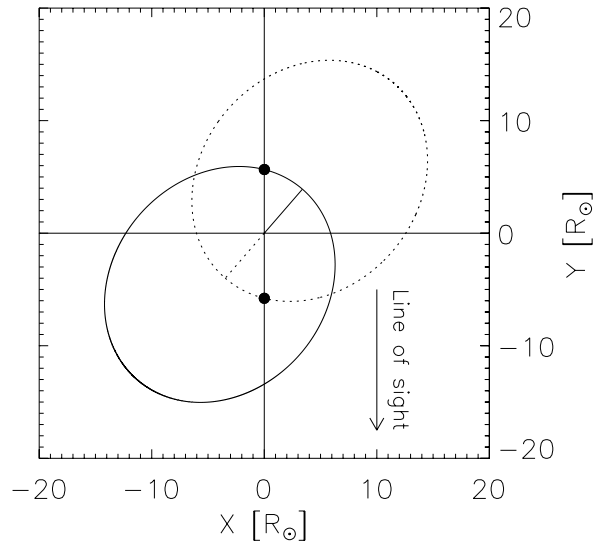

Fig. 5. The orbit of the binary system V1143 Cyg shown from above the orbital plane. The solid line represents the orbit of the primary component and the dashed line the orbit of the secondary. The lines from the center of gravity towards the orbits indicate the position of the periastron. The big dots indicate the positions of the stars at time of mid primary eclipse. exposure. This artificially widens a BF taken during a 20 min exposure relative to a $\mathrm{BF}$ which would have been taken instantaneously. During the eclipses, a second effect becomes important; the coverage of the eclipsed stars can change considerably during 20-min. Again, this effect is stronger during the primary eclipse than during the secondary eclipse, as the primary eclipse is deeper and shorter. Sine we need an exposure over several minutes to receive a sufficiently high $\mathrm{S} / \mathrm{N}$ ratio $^{3}$, we introduced the same "smearing" in the simulated BFs. We calculated three BFs for each exposure and stacked them together: one simulated observation $6.6 \mathrm{~min}$ before the photon midpoint, one at the photon midpoint and one $6.6 \mathrm{~min}$ after the photon midpoint. We convolved each simulated BF with a Gaussian of $\sigma=2.5 \mathrm{~km} \mathrm{~s}^{-1}$, representing roughly the resolution of the spectrograph in the wavelength range used.

In addition to the parameters describing the binary system, one parameter is included that scales all simulated BFs to the observed BFs. The primary star has a slightly higher luminosity than the secondary. Also, the template used might fit one of the two stars better than the other one. Therefore, we included another parameter scaling the height of the kernels of the two stars relative to each other. As mentioned in the beginning of this section, all spectra have been normalized. During the eclipses the absolute amount of light changes. Hence the depths of the absorption lines change relative to the normalized continuum, and therefore the height of the BFs also change. This has to be incorporated in the calculations of the BFs. We performed a fit using all 46 spectra obtained out of eclipse and during the primary and secondary eclipses. The derived values for the parameters are

\footnotetext{
${ }^{3}$ Out of eclipse the spectra have a S/N between 40 and 50 .
}

given in column four of Table 2. The measured BFs and the best fits can be seen in Fig. 8 for the primary eclipse and in Fig. 9 for the secondary eclipse. The uncertainties were calculated using the bootstrap method described in Press et al. (1992).

\section{Discussion}

\subsection{Orbital parameters}

Using the methods described in Sects. 3.1 and 3.2 we obtained values for the orbital parameters of V1143 Cyg and for the stellar parameters of the two stars. The time of periastron passage of the primary $(T)$, the longitude of the periastron $(\omega)$, and the eccentricity $(e)$ were determined in all three fits, and agree with each other to within the $1-\sigma$ level. Using the values from Giménez $\&$ Margrave $(1985)$ for $\omega\left(48.26 \pm 0.01^{\circ}\right)$ and the apsidal motion rate $(0.000705 \pm 0.000041 \%$ cycle $)$, and taking the time difference into account, one would derive an $\omega$ of $49.31^{\circ} \pm 0.06^{\circ}$ for the time of our observations. This is also the value stated in Table 2. The parameter for the semi-amplitude of the secondary $\left(K_{\mathrm{s}}\right)$ $\left(90.0 \pm 0.1 \mathrm{~km} \mathrm{~s}^{-1}\right)$ falls outside the $1-\sigma$ range of the literature value $\left(91.1 \pm 0.4 \mathrm{~km} \mathrm{~s}^{-1}\right)$. This difference can also be seen in the values for the projected semi-major axis of the system $(a \sin i)$. The uncertainty in the radial-velocity of the center of gravity of the system $(\gamma)$ includes the uncertainty of the radial-velocity in our template star, HD 222368 (5.6 $\pm 0.3 \mathrm{~km} \mathrm{~s}^{-1}$, Udry et al. 1999). Our calculated masses for the two components in V1143 Cyg are $M_{\mathrm{p}}=1.355 \pm 0.004 M_{\odot}$ and $M_{\mathrm{s}}=1.327 \pm 0.003 M_{\odot}$. These values lie in between the values calculated by Andersen et al. (1987) $M_{\mathrm{p}}=1.391 \pm 0.016 M_{\odot}$ and $M_{\mathrm{S}}=1.347 \pm 0.013 M_{\odot}$, and the 


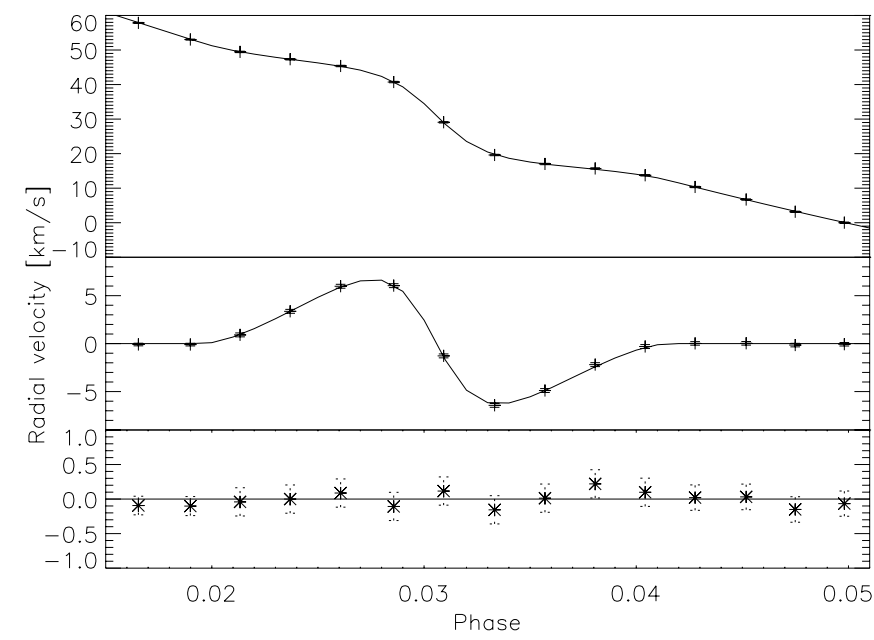

Fig. 6. The rotation effect during the primary eclipse. The shift of the center of gravity is plotted against the orbital phase. The upper panel shows the measured radial velocities along with the best fit. In the second panel the radial velocity due to the orbital motion is subtracted from the data and the fit. The residuals between data and fit are shown in the third panel.

values given by Giménez \& Margrave (1985) $M_{\mathrm{p}}=1.33 \pm$ $0.03 M_{\odot}$ and $M_{\mathrm{s}}=1.29 \pm 0.03 M_{\odot}$.

\subsection{Stellar parameters}

The stellar parameters obtained in Sect. 3.1 are derived by analyzing the shape of the rotation anomaly in the radial velocity. The method relies on a clean subtraction of the foreground star from the obtained spectra before calculating the BF's, which depend on orbital parameters derived from out-of-eclipse measurements, values for the stellar radii, the stellar limb-darkening and the light ratio between the two stars during the subtraction process, which have been taken from the literature.

In Sect. 3.2, the change in the shape of the absorption lines is used instead of the rotation anomaly. Looking at Figs. 8 and 9, one can see that the simulated BFs are somewhat "rounder" during eclipses than the observed BFs. This can clearly be seen during the central phase of the primary eclipse (Fig. 8 panels in the second row) and during the secondary eclipse (Fig. 9).

The agreement between the measured BF and the observed $\mathrm{BF}$ can be improved if $u$ is included in the fit. The derived values for $u$ would be $0.9 \pm 0.1$ for the primary and $0.8 \pm 0.1$ for the secondary. As these values are probably too high (Gray 2005) and the derived values for $\beta$ would not change significantly $\left(\beta_{\mathrm{p}}=\right.$ $-0.6 \pm 1.4^{\circ}$ and $\beta_{\mathrm{s}}=-0.3 \pm 1.2^{\circ}$ ), we kept $u$ fixed to 0.6 in the final fits.

Including solar-like differential rotation, the orbital inclination and the stellar radii as free parameters in the fits also leads to a better agreement between data and simulation. However, the derived differential rotation parameters are negative for both stars; the angular rotation speed is faster at the poles than at the equator. Furthermore, the fitted radii are not in agreement with the literature values; the primary radius is increased relative to the literature value and the secondary decreased relative to its literature value. The value for the orbital inclination of V1143 Cyg in the literature is reproduced by our fit, but weakly constrained. A negative differential rotation parameter would give the BFs during the eclipses a more box-like shape. A bigger difference between the stellar radii would further improve the agreement between simulation and data during the central part of the

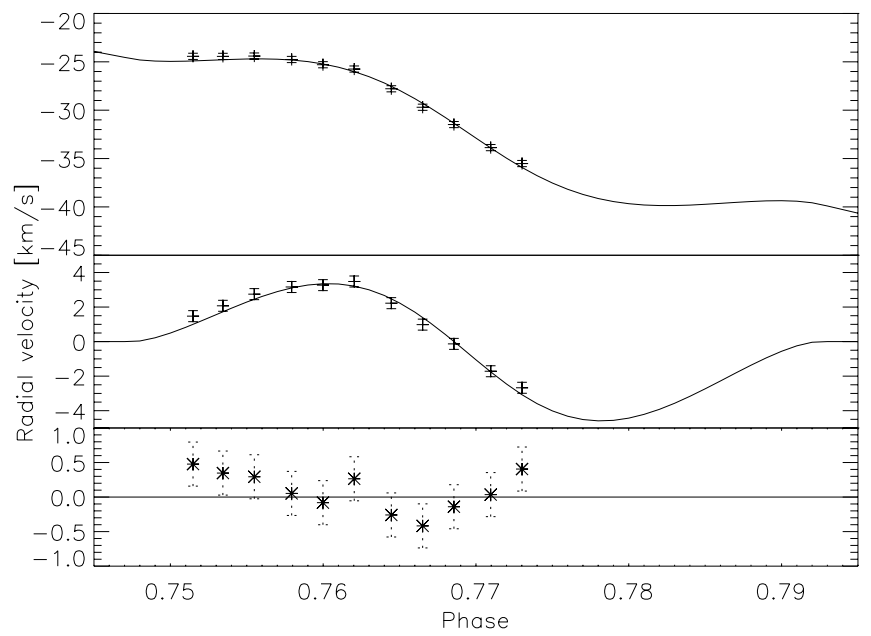

Fig. 7. The same as for Fig. 6 but this time for the secondary eclipse.

primary eclipse. However, a bigger difference in stellar radii would also mean a bigger difference in stellar masses, which would not agree with the nearly equal semi-amplitudes found in the radial-velocity.

We favor a different explanation for the difference of "roundness" between the measured and simulated BFs. Looking at an absorption line originating from one small area of the stellar disk (i.e. no rotational broadening), the core of an absorption line is formed higher up in the stellar atmosphere than the wings (see Gray 2005), as the same optical depth is reached earlier in the core than in the wings of the absorption line. One can now compare the effect that limb-darkening has on the core and the wings of the absorption lines. In the core of an absorption line, a high optical depth is reached in a high layer of the atmosphere looking down on a stellar atmosphere. This also means that looking from an angle into the stellar atmosphere (i.e. at the limb) will result in a small change in the average height at which the core of the line is formed: only the outer cooler parts of the atmosphere are probed. For the wings of the absorption lines the situation is different; the height at which the wings of the observed absorption line are formed changes more from the center to the limb of the stellar disk. Therefore, in going from the center to the limb of a stellar disk the absorption lines become more box-like. The particular shape change of an absorption line originating under different angles relative to the observer depends on the details of the stellar atmosphere. This would result in a non-linear limbdarkening law. Out of eclipse, the light is integrated over the whole stellar surface, but for example, during the central part of the primary eclipse light is only received from the outer part of the stellar disk (see Fig. 8, icon in the panel of the third column and second row). Therefore, the shape change of the absorption lines originating from different parts of the stellar disk might explain the difference between the observed BFs and the simulated BFs (e.g., Pierce \& Slaughter 1982; Balthasar 1988; or Hadrava 2007).

Using the broadening of about 1000 lines, we obtained macro-turbulence parameters $\left(\zeta_{\mathrm{RT}}\right)$ of $3.4 \mathrm{~km} \mathrm{~s}^{-1}$ and $3.3 \mathrm{~km} \mathrm{~s}^{-1}$ for the primary and secondary, respectively. For comparison, the macro-turbulence parameter found for the Sun, as a disk-integrated star, for weak and moderately strong lines is $\approx 4.0 \mathrm{~km} \mathrm{~s}^{-1}$ (Takeda 1995).

The values obtained for the $v \sin i$ of the secondary, using the two different methods described above, agree with each other and are also consistent with the literature value. Note that there 

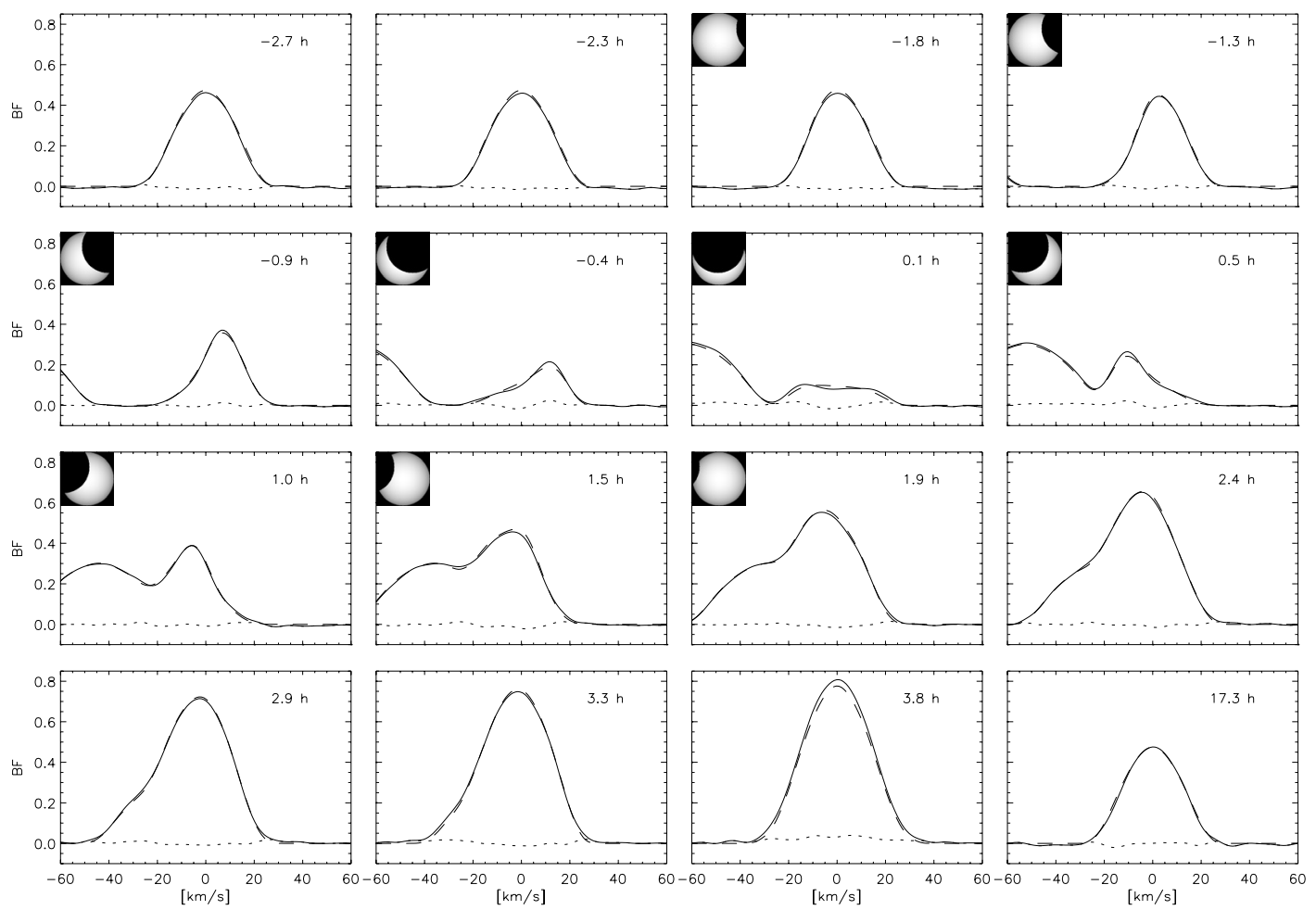

Fig. 8. BFs during the primary eclipse centered in the frame of the primary. Each panel represents one measurement. The solid line represents the measured BF, the dashed line shows the best fit BF, while the dotted line represents the difference between the fit and the measurement. The numbers in the upper right corner of each observation indicate the time difference between the photon midpoint of the observation and the midpoint of the eclipse in hours. In the upper left corner one can see the uncovered part of the primary half-sphere for the photon midpoint of that measurement if the star was eclipsed during this measurement. One can see that the BF of the secondary moves closer to the primary BF in velocity space over the course of the eclipse. Please note that the scale of the ordinate is arbitrary. The panel in the lower right corner shows the $\mathrm{BF}$ of the primary for an observation out of eclipse.
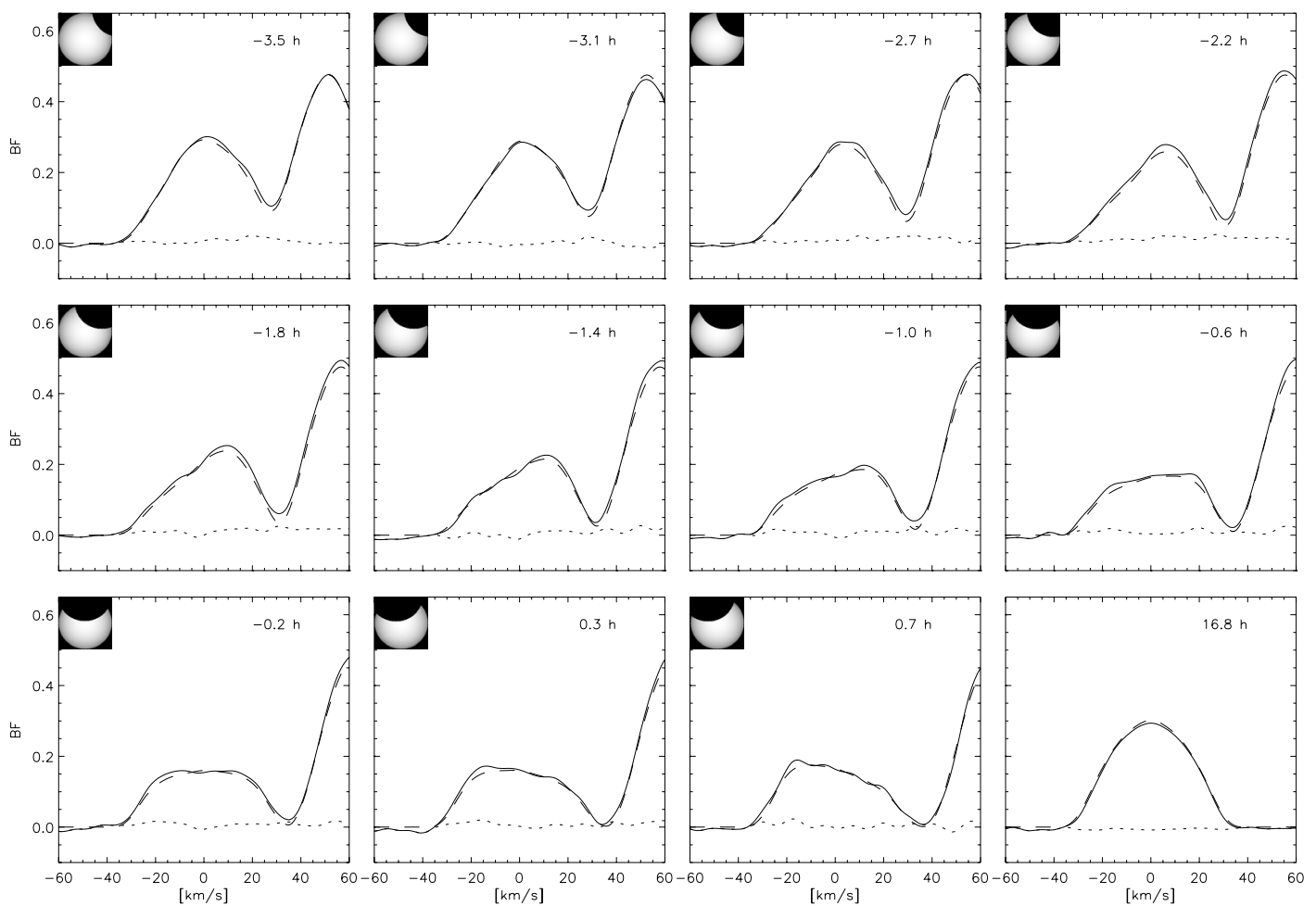

Fig. 9. The same as Fig. 8 but for the secondary eclipse. One can see that the BF of the primary moves further away from the secondary BF in velocity space over the course of the eclipse. The panel in the lower right corner shows the BF of the secondary for an observation out of eclipse. 
Table 2. Derived parameters of V1143 Cyg are given together with their formal errors. The reduced $\chi^{2}$ of the orbit fit is 0.95 and the reduced $\chi^{2}$ of the joint fit is 0.96 . The average uncertainty in radial-velocity for the primary is $0.16 \mathrm{~km} \mathrm{~s}^{-1}$ and $0.27 \mathrm{~km} \mathrm{~s}^{-1}$ for the secondary, in the joint fit. For comparison, values given by Andersen et al. (1987) and Giménez \& Margrave (1985) are also shown. The second and third columns present the parameters obtained in Sect. 3.1. The fourth column shows the parameters derived with the method presented in Sect. 3.2. For the reasons mentioned in Sect. 4 we consider the values found in Sect. 3.2 to be our best parameters and the formal errors given for $v \sin i$ and $\zeta_{\mathrm{RT}}$ as too small.

\begin{tabular}{|c|c|c|c|c|}
\hline \multirow[t]{2}{*}{$\overline{\text { Parameter }}$} & \multicolumn{2}{|c|}{ Center } & \multirow[t]{2}{*}{ Shape } & \multirow{2}{*}{ 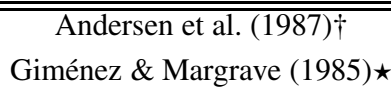 } \\
\hline & Orbit & Joint fit & & \\
\hline$T$ [JD-2 400000$]$ & $53536.130 \pm 0.002$ & $53536.131 \pm 0.002$ & $53536.1317 \pm 0.0006$ & \\
\hline$K_{\mathrm{p}}\left[\mathrm{km} \mathrm{s}^{-1}\right]$ & $88.1 \pm 0.04$ & $88.1 \pm 0.1$ & $88.01 \pm 0.05$ & $88.2 \pm 0.2 \dagger$ \\
\hline$K_{\mathrm{s}}\left[\mathrm{km} \mathrm{s}^{-1}\right]$ & $90.1 \pm 0.08$ & $90.1 \pm 0.2$ & $89.9 \pm 0.1$ & $91.1 \pm 0.4 \dagger$ \\
\hline$e$ & $0.538 \pm 0.001$ & $0.538 \pm 0.001$ & $0.5378 \pm 0.0003$ & $0.540 \pm 0.003 \dagger$ \\
\hline$\omega\left[^{\circ}\right]$ & $49.1 \pm 0.2$ & $49.1 \pm 0.2$ & $49.27 \pm 0.05$ & $49.31 \pm 0.06 \star$ \\
\hline$a \sin i\left[R_{\odot}\right]$ & $22.67 \pm 0.03$ & $22.67 \pm 0.03$ & $22.64 \pm 0.02$ & $22.78 \pm 0.08 \dagger$ \\
\hline$\gamma\left[\mathrm{km} \mathrm{s}^{-1}\right]$ & $-16.8 \pm 0.3$ & $-16.8 \pm 0.3$ & $-16.8 \pm 0.3$ & $-16.5 \pm 0.7 \dagger$ \\
\hline$M_{\mathrm{p}} \sin ^{3} i\left[M_{\odot}\right]$ & $1.357 \pm 0.005$ & $1.357 \pm 0.008$ & $1.350 \pm 0.004$ & $1.386 \pm 0.016 \dagger$ \\
\hline$M_{\mathrm{s}} \sin ^{3} i\left[M_{\odot}\right]$ & $1.327 \pm 0.004$ & $1.327 \pm 0.007$ & $1.322 \pm 0.003$ & $1.341 \pm 0.013 \dagger$ \\
\hline$v \sin i_{\mathrm{p}}\left[\mathrm{km} \mathrm{s}^{-1}\right]$ & & $16.9 \pm 1.0$ & $19.6 \pm 0.1$ & $18 \pm 2 \dagger$ \\
\hline$v \sin i_{\mathrm{s}}\left[\mathrm{km} \mathrm{s}^{-1}\right]$ & & $28.0 \pm 5.0$ & $28.2 \pm 0.1$ & $27 \pm 3 \dagger$ \\
\hline$\zeta_{\mathrm{RT}} P\left[\mathrm{~km} \mathrm{~s}^{-1}\right]$ & & & $3.4 \pm 0.1$ & \\
\hline$\zeta_{\mathrm{RT}} S\left[\mathrm{~km} \mathrm{~s}^{-1}\right]$ & & & $3.3 \pm 0.1$ & \\
\hline$\beta_{\mathrm{p}}\left[{ }^{\circ}\right]$ & & $0.5 \pm 4.0$ & $0.3 \pm 1.5$ & \\
\hline$\beta_{\mathrm{s}}\left[{ }^{\circ}\right]$ & & $-3.9 \pm 4.0$ & $-1.2 \pm 1.6$ & \\
\hline
\end{tabular}

might be a systematic difference between the obtained radialvelocities of the secondary during the secondary eclipse and the fit to the radial-velocities (Fig. 7). This might be caused by too high an uncertainty in the orbital parameters used during the data analysis in Sect. 3.1. The values obtained for the primary $v \sin i$ do agree with the literature value within their $1-\sigma$ error, but do not agree with each other within their $1-\sigma$ errors. The method applied in Sect. 3.1 uses the amplitude of the RM effect to derive the value for the projected rotational velocity. It assumes a linear limb-darkening coefficient. As mentioned in the last paragraph, this might not be sufficient. Also in Sect. 3.2 a linear limb-darkening coefficient is used, but $v \sin i$ is derived not only by the use of the RM effect, but also by the shape of the BF outside of the eclipse. Therefore, we disregard the value for $v \sin i$ obtained in Sect. 3.1 and take the value of $19.6 \mathrm{~km} \mathrm{~s}^{-1}$ from Sect. 3.2 as the projected rotational velocity for the primary star in V1143 Cyg. Due to the remaining mismatch between the data and the simulation, which might be due to the use of too simple a model, we consider the formal errors for $\zeta_{\mathrm{RT}}$ and $v \sin i$ as too small.

We would like to point out that the methods used are still in their infancy. Even with the derived values for the stellar radii, the orbital inclination, limb-darkening and differential rotation are not free of errors yet; these parameters are not normally accessible, or only with great difficulty, via spectroscopic data.

\subsection{Orientation of the rotation axes}

The main focus of this work is the robust determination of the orientation of the stellar rotation axes. The values derived for the projection of the rotation axes onto the plane of the sky are given in the last two rows of Table 2 . The $\beta$ s derived from the two different methods agree to within their errors despite the different systematic problems of each method. Therefore, the projections of the rotation axes of both stars are, to within their uncertainties, perpendicular to a vector that lies in the plane of the orbit and is perpendicular to the line of sight. What does that mean for the orientation axes of the stars? It is highly unlikely that the geometry is such that we see the projection of the rotation axes perpendicular to the orbital plane and the stellar rotation axes have a high inclination towards the observer. We therefore conclude that the stellar rotation axes are normal to the orbital plane, and aligned with each other and the rotation axis of the system.

This leaves the theoretical prediction of the apsidal motion unaltered for V1143 Cyg. Hence, the difference between expected $(0.00089 \pm 0.00015 \%$ cycle $)$ and measured apsidal motion $(0.000705 \pm 0.000041 \%$ cycle $)$, which just lies outside the $1-\sigma$ error bars, is also unchanged. The effect of a misalignment between the stellar rotation axes and the orbital spin axis on the apsidal motion has been studied by Kopal (1978), Shakura (1985), Company et al. (1988), and Petrova \& Orlov (2003). The contribution of the stellar rotation to the advance of the longitude of the periastron is reduced if the stellar rotation axis is tilted against the orbit spin axis until finally, when the axis of stellar rotation lays in the orbital plane, its contribution is half as large and with the opposite sign as when the stellar and orbital axes would be parallel. In this situation, it contributes to a retrograde rotation of the periastron. The contribution of the stellar rotation to the apsidal motion depends not only on the orientation of the axis, but also on the square of the angular stellar rotation rate. As one measures only $v \sin i$, a greater inclination towards the observer would mean a higher angular stellar rotation rate, and therefore a greater contribution of the rotation term to the overall apsidal motion. We calculate that if the rotation axes of both stars would lie in the orbital plane, but have no inclination towards the observer, then the complete apsidal motion would be $0.00073^{\circ}$ cycle. If the rotation axes would have an inclination towards the observer of $i \approx 70^{\circ}$ in either of the two stars, or of $i \approx 60^{\circ}$ in both stars, only then would the expected and measured apsidal motion be in agreement. The secondary, due to its higher $v \sin i$, has a larger influence on the rotational term of the apsidal motion than the primary component. As already pointed out, it is very unlikely that the stellar and orbital spin axes span a large angle, while their projections on the sky are, in their uncertainties, parallel.

Our findings do not support the hypothesis advocated by Petrova \& Orlov (2003), that a misalignment of the stellar rotation axes with the orbital spin could bring the theoretical and measured apsidal motion for a number of binary systems, including V1143 Cyg, into better agreement. 
Our work has excluded the option of a misalignment between the stellar rotation axes as a possible explanation for the difference between the expected and measured apsidal motion in V1143 Cyg. It is therefore interesting to look at other possibilities that might explain this difference. As the apsidal motion constant $\left(k_{2}\right)$ is an important source of uncertainty in the calculation of the expected apsidal motion, a new calculation of the apsidal motion constant for V1143 Cyg using modern codes for stellar evolution might be of value.

In our analysis of the orbital data we found no indication of a third body in the V1143 Cyg system, whose influence might also alter the apsidal motion. However, because of the limited coverage in time (one year) and the limited accuracy in radial-velocity, combined with the possibility that the orbit of a third body could have a lower inclination, a third body cannot be excluded.

The alignment of the stellar rotation axes could also set a lower limit to the age of the system, if the axes were not aligned at the birth of the system. However, this is not a straightforward argument, since, during the pre-main-sequence phase, synchronizing forces must have been larger, due to the larger sizes of the stars. Proper modeling of the evolution of V1143 Cyg might reveal whether it had enough time to align its axes. If the timespan to align the axes is longer than the lifetime of the system, it would mean that V1143 Cyg, with its high eccentricity of 0.54 , was born in this way, with all spin and orbital axes aligned.

\section{Conclusions}

We measured the Rossiter-McLaughlin effect for both components in the binary system V1143 Cyg. We developed two different methods to derive the angle $\beta$ between the stellar spin axis projected onto the plane of the sky and the orbital spin axis projected onto the plane of the sky.

Using the first method, i.e. by determination of the center of the broadening function, we showed how it is possible to subtract the absorption lines of the foreground star during an eclipse from the spectrum. This made it possible to use the shift of the center of gravity of the absorption lines as a proxy for the rotation effect, even in systems with blending occuring during eclipses, and derive values for $\beta$ and $v \sin i$. However, for systems with low eccentricity, blending of the spectral lines during eclipses is stronger than in V1143 Cyg. Therefore, every systematic error in the tomography or in the subtraction of the foreground spectrum due to the parameters used in the subtraction, will have a substantial effect on the value of $\beta$ derived.

The second method presented in this paper, the modeling of the shape of the broadening functions, avoids the problems of the first method by taking the influence of the eclipsing star into account explicitly. This makes the method more suitable for eclipsing binary systems with low eccentricity (higher blending of the spectral lines). The use of the complete BFs, instead of the center of gravity or the Gaussian fit to the line, has the additional advantage that it makes the fitting of the parameters $\beta$ and $v \sin i$ more precise. However, the derived values for these parameters are not necessarily free of systematic errors. Further work is needed here to make it possible to derive information about velocity fields on the stellar surface represented by macro-turbulence, or differential rotation. If differential rotation is present it might be possible to derive some information about the inclination of the rotation axes towards the observer.

With both methods we determined orbital and stellar parameters which agree within their uncertainties with values derived in earlier studies, with the exception of the semi-amplitude of the secondary. The values derived for the angle $\beta$ agree with each other. We found that the stellar rotation axes in V1143 Cyg are normal to the orbital plane for both components, which leaves the theoretical predictions for the apsidal motion unchanged. This makes V1143Cyg the first binary system with main-sequence stars for which the orientations of the rotation axes of both components are determined.

Even with progress made in the field of apsidal motion in the last years (e.g. Claret \& Willems 2002), it is interesting to apply the methods discussed here to other binary systems where there is a stronger disagreement between the observed and expected apsidal motion (e.g. DI Herculis, Claret 1998), in order to investigate whether a misalignment of the stellar rotation axes can be excluded as a cause for the disagreement, or a misalignment contributes to the difference. Furthermore, information about the orientation of the stellar rotation axes in binary systems could be used to explore the dependence of the synchronization time scales on the semi-major axis, eccentricity of the binary system and the stellar type of its components. This might lead to new insight about the formation and evolution of binary systems and stellar interiors.

Acknowledgements. We kindly thank Saskia Hekker for taking part of the observations used in this study, as well as the staff at the Lick Observatory for their excellent support during the observations. We would also like to thank Craig Markwardt for the use of routines taken from his web page at http://cow.physics.wisc.edu/ craigm/idl/idl.html.

This research has made use of the Simbad database located at http://simbad.u-strasbg.fr/ and the Vienna Atomic Line database (VALD) located at http://ams .astro.univie.ac.at/vald/.

\section{References}

Andersen, J., Nordstrom, B., Garcia, J. M., \& Giménez, A. 1987, A\&A, 174, 107 Bagnuolo, Jr., W. G., \& Gies, D. R. 1991, ApJ, 376, 266

Balthasar, H. 1988, A\&AS, 72, 473

Claret, A. 1998, A\&A, 330, 533

Claret, A., \& Willems, B. 2002, A\&A, 388, 518

Company, R., Portilla, M., \& Gimenez, A. 1988, ApJ, 335, 962

ESA. 1997, ESA SP-1200

Giménez, A. 2006, ApJ, 650, 408

Giménez, A., \& Margrave, T. E. 1985, AJ, 90, 358

Gray, D. F. 2005, The Observation and Analysis of Stellar Photospheres, 3rd edn., ed. D. F. Gray (Cambridge, UK: Cambridge University Press)

Hadrava, P. 2007, in Solar and Stellar Physics Through Eclipses, ed. O. Demircan, S. O. Selam, \& B. Albayrak, ASP Conf. Ser., 370, 164 Hosokawa, Y. 1953, PASJ, 5, 88

Hube, D. P., \& Couch, J. S. 1982, Ap\&SS, 81, 357

Kopal, Z. 1959, Close binary systems, The International Astrophysics Series (London: Chapman \& Hall)

Kopal, Z. 1978, Astrophysics and Space Science Library, Vol. 68, Dynamics of Close Binary Systems

McLaughlin, D. B. 1924, ApJ, 60, 22

Ohta, Y., Taruya, A., \& Suto, Y. 2005, ApJ, 622, 1118

Petrova, A. V., \& Orlov, V. V. 2003, Astrophysics, 46, 329

Pierce, A. K., \& Slaughter, C. 1982, ApJS, 48, 73

Press, W. H., Teukolsky, S. A., Vetterling, W. T., \& Flannery, B. P. 1992, Numerical recipes in FORTRAN. The art of scientific computing (Cambridge: University Press), 2nd edn.

Queloz, D., Eggenberger, A., Mayor, M., et al. 2000, A\&A, 359, L13

Rossiter, R. A. 1924, ApJ, 60, 15

Rucinski, S. 1999, in Precise Stellar Radial Velocities, ed. J. B. Hearnshaw, \& C. D. Scarfe, IAU Colloq. 170, ASP Conf. Ser., 185, 82

Shakura, N. I. 1985, Sov. Astron. Lett., 11, 224

Snellen, I. A. G. 2004, MNRAS, 353, L1

Takeda, Y. 1995, PASJ, 47, 337

Udry, S., Mayor, M., Maurice, E., et al. 1999, in Precise Stellar Radial Velocities, ed. J. B. Hearnshaw, \& C. D. Scarfe, IAU Colloq. 170, ASP Conf. Ser., 185, 383

Winn, J. N., Johnson, J. A., Marcy, G. W., et al. 2006, ApJ, 653, L69

Worek, T. F. 1996, PASP, 108, 962 
S. Albrecht et al.: Spin axes in V1143 Cyg, Online Material p 1

\section{Online Material}




\section{Appendix A: Data}

Table A.1. Radial velocity measurements of V1143 Cyg out of eclipse and during the primary and secondary eclipse. The photon midpoints of the observations are given in the first column, and the radial velocities of the primary and secondary are given in the second and third column.

\begin{tabular}{|c|c|c|}
\hline HJD & Vel. P & Vel. S \\
\hline [JD-2 400000$]$ & {$\left[\mathrm{km} \mathrm{s}^{-1}\right]$} & {$\left[\mathrm{km} \mathrm{s}^{-1}\right]$} \\
\hline 53612.665 & $35.40 \pm 0.13$ & $-81.71 \pm 0.22$ \\
\hline 53612.683 & $30.58 \pm 0.14$ & $-76.43 \pm 0.23$ \\
\hline 53651.654 & $-71.40 \pm 0.14$ & $27.82 \pm 0.22$ \\
\hline 53613.663 & $-76.90 \pm 0.14$ & $33.40 \pm 0.23$ \\
\hline 53613.771 & $-78.44 \pm 0.14$ & $34.56 \pm 0.22$ \\
\hline 53613.880 & $-79.27 \pm 0.14$ & $35.48 \pm 0.23$ \\
\hline 53934.843 & $-79.49 \pm 0.14$ & $35.83 \pm 0.23$ \\
\hline 53583.676 & $-78.86 \pm 0.14$ & $35.39 \pm 0.23$ \\
\hline 53936.818 & $-57.40 \pm 0.13$ & $13.47 \pm 0.22$ \\
\hline 53585.669 & $-51.69 \pm 0.15$ & $6.91 \pm 0.25$ \\
\hline 53649.639 & $43.23 \pm 0.14$ & $-89.43 \pm 0.23$ \\
\hline 53611.709 & $62.33 \pm 0.14$ & $-109.40 \pm 0.23$ \\
\hline 53611.728 & $63.65 \pm 0.14$ & $-111.21 \pm 0.24$ \\
\hline 53611.814 & $70.69 \pm 0.13$ & $-117.65 \pm 0.22$ \\
\hline 53611.835 & $72.30 \pm 0.14$ & $-119.47 \pm 0.23$ \\
\hline 53611.884 & $76.29 \pm 0.14$ & $-123.12 \pm 0.23$ \\
\hline 53611.903 & $77.69 \pm 0.13$ & $-125.03 \pm 0.22$ \\
\hline 53932.846 & $80.45 \pm 0.13$ & $-127.61 \pm 0.22$ \\
\hline 53581.675 & $96.71 \pm 0.14$ & $-144.12 \pm 0.24$ \\
\hline 53650.612 & $88.17 \pm 0.14$ & $-135.32 \pm 0.23$ \\
\hline 53650.737 & $67.50 \pm 0.14$ & $-113.86 \pm 0.22$ \\
\hline 53612.865 & $-12.10 \pm 0.18$ & $-33.23 \pm 0.27$ \\
\hline 53612.884 & $-15.74 \pm 0.19$ & $-29.58 \pm 0.28$ \\
\hline 53612.901 & $-19.29 \pm 0.18$ & $-26.26 \pm 0.28$ \\
\hline 53612.919 & $-22.44 \pm 0.18$ & $-22.26 \pm 0.27$ \\
\hline 53579.678 & $-11.09 \pm 0.18$ & $-33.60 \pm 0.28$ \\
\hline 53612.701 & $27.01 \pm 0.20$ & \\
\hline 53612.719 & $24.88 \pm 0.20$ & \\
\hline 53612.737 & $22.93 \pm 0.20$ & \\
\hline 53612.757 & $18.28 \pm 0.20$ & \\
\hline 53612.775 & $6.63 \pm 0.20$ & \\
\hline 53612.793 & $-2.84 \pm 0.20$ & \\
\hline 53612.811 & $-5.44 \pm 0.20$ & \\
\hline 53612.829 & $-6.75 \pm 0.20$ & \\
\hline 53612.847 & $-8.71 \pm 0.20$ & \\
\hline 53587.717 & & $-46.88 \pm 0.32$ \\
\hline 53587.732 & & $-46.88 \pm 0.32$ \\
\hline 53587.748 & & $-46.85 \pm 0.32$ \\
\hline 53587.767 & & $-47.20 \pm 0.32$ \\
\hline 53587.782 & & $-47.74 \pm 0.32$ \\
\hline 53587.798 & & $-48.19 \pm 0.32$ \\
\hline 53587.816 & & $-50.23 \pm 0.32$ \\
\hline 53587.832 & & $-52.14 \pm 0.32$ \\
\hline 53587.848 & & $-53.93 \pm 0.32$ \\
\hline 53587.866 & & $-56.31 \pm 0.32$ \\
\hline 53587.882 & & $-57.96 \pm 0.32$ \\
\hline
\end{tabular}

